Vietnam Journal of Mechanics, VAST, Vol.38, No. 4 (2016), pp. 249 - 265

DOI:10.15625/0866-7136/6954

\title{
FREE VIBRATION ANALYSIS OF JOINED COMPOSITE CONICAL-CYLINDRICAL-CONICAL SHELLS CONTAINING FLUID
}

\author{
Vu Quoc Hien ${ }^{1}$, Tran Ich Thinh ${ }^{2, *}$, Nguyen Manh Cuong ${ }^{2}$ \\ ${ }^{1}$ Viet Tri University of Industry, Phu Tho, Vietnam \\ ${ }^{2}$ Hanoi University of Science and Technology, Vietnam \\ *E-mail: tranichthinh@yahoo.com \\ Received September 12, 2015
}

\begin{abstract}
A new continuous element (CE) formulation has been presented in this paper for the vibration analysis of cross-ply composite joined conical-cylindrical-conical shells containing fluid. Governing equations are obtained using thick shell theory of Midlin, taking into account the shear deflection effects. The velocity potential, Bernoulli's equation and impermeability condition have been applied to the shell-fluid interface to obtain an explicit expression for fluid pressure. The dynamic stiffness matrix has been built from which natural frequencies have been calculated. The appropriate expressions among stress resultants and deformations are extracted as continuity conditions at the joining section. A matlab program is written using the CE formulation in order to validate our model. Numerical results on natural frequencies are compared to those obtained by the Finite Element Method and validated with the available results in other investigations. This paper emphasizes advantages of CE model, the effects of the fluid filling and shell geometries on the natural frequencies of joined composite conical-cylindrical-conical shells containing fluid.
\end{abstract}

Keywords: Free vibration, cross-ply composite joined conical-cylindricall-conical shells, dynamics stiffness matrix, continuous element method.

\section{INTRODUCTION}

The joined shells filled with fluid of revolution have many applications in various branches of engineering such as mechanical, aeronautical, marine, civil and power engineering. Hence, the comprehension of dynamic behaviours of such structures is of great important in order to design and fabric safer and more economic composite shell structures.

There are many computational methods available for the free vibration of the cylindrical and conical and joined cylindrical-conical shells, such as the exact wave solution,

(C) 2016 Vietnam Academy of Science and Technology 
Ritz method, Gelerkin method, differential quadrature method, state space method, finite difference method, Finite Element Method (FEM) and Continuous Element Method (CEM) or Dynamic Stiffness Method (DSM). Sivadas and Ganesan [1] investigated the effects of thickness variation on natural frequencies of laminated conical shells by a semianalytical finite element method. Xi, Yam and Leung [2,3] analyzed the free vibration of a laminated composite circular cylindrical shell partially filled with fluid using a semianalytical finite element technique based on the Reissner-Mindlin theory and compressible fluid equations. Katsutoshi et al. [4] analyzed the free vibrations of a laminated composite circular cylindrical shell partially filled with liquid. Toorani and Lakis [5] studied the effect of shear deformation in the dynamic behaviour of anisotropic laminated open cylindrical shells filled with fluid. Kochupillai, Ganesan and Padmanabhan [6] performed a dynamic analysis of composite shells conveying fluid based on semi-analytical coupled finite element formulation Larbi et al. [7] presented the theoretical and finite element formulations of piezoelectric composite shells of revolution filled with compressible fluid A semi-analytical approach has been utilized by Toorani and Lakis [8] to determine the swelling effect on the dynamic behaviour of composite cylindrical shells conveying fluid. Tong $[9,10]$ proposed the power series expansion approach to study the free vibration of orthotropic composite laminated conical shells. Shu [11] has employed the differential quadrature method to study the vibration of conical shells. The investigations of vibration analysis for composite cylindrical shells are carried out by using different approaches such as 2D finite element model based on classical thin shell theory [12], 2D analytical method using the cubic spline functions [13], analytical method based on the first-order shear deformation theory (FSDT) [14]. Senthil and Ganesan [15] performed a dynamic analysis of composite conical shells filled with fluid. Kerboua, Lakis and Hmila [16] using a combination of finite element method and classical shell theory to determine the natural frequencies of anisotropic truncated conical shells in interaction with fluid.

Irie et al. [17] used the transfer matrix approach to study the free vibration of joined isotropic conical-cyclindrical shells. Patel et al. [18] presented some vibrational results for laminated composite joined conical-cyclindrical shell with first order shear deformation theory using finite element method (FEM). Recently, Caresta and Kessissoglou [19] analyzed the free vibrations of joined truncated conical-cyclindrical shells. The displacements of the conical sections were solved using a power series solution, while a wave solution was used to describe the displacements of the cylindrical sections. Both DonnellMushtari and Flugge equations of motion were used. Kouchakazadeh and Shakouri [20] studied the vibrational behaviour of two joined cross-ply laminated conical shells, joined cylindrical-conical shells. Governing equations are obtained using thin-walled shallow shell theory of Donnell type and Hamilton's principle. The appropriate expressions among stress resultants and deformations are extracted as continuity condition at the joining section of the cones. 
In those studies, low natural frequencies are generally investigated. The known disadvantage of traditional methods like FEM is the discretization operation of the domain which causes errors in dynamic analysis, especially in medium and high frequencies. For medium and high frequency range, the CEM can be applied with many advantages: high precision, rapid calculating speed, reduction of the model size and of the computing time. Numerous Continuous Elements have been established for metal and composite beams [21,22] and plates [23]. Nguyen Manh Cuong and Casimir [24] have succeeded in building the DSM for thick isotropic plate and shells of revolution. The CE models for composite cylindrical shells and conical shells presented in works of Tran Ich Thinh and Nguyen Manh Cuong $[25,26]$ imposed a considerable advancement of the study on CEM for metal and composite structures. Recently, the new research for thick laminated composite joined cylindrical-conical shells by Tran Ich Thinh, Nguyen Manh Cuong and Vu Quoc Hien [27] has emphasized the strong capacity of CEM in assembling complex structure.

The main objective of this paper is to present a detailed study on free vibrations of a composite conical-cylindrical-conical shells containing an incompressible and inviscid liquid. Illustrative examples are provided to demonstrate the accuracy and efficiency of the developed numerical procedure.

\section{FORMULATION OF JOINED CROSS-PLY COMPOSITE CONICAL-CYLINDRICAL-CONICAL SHELLS CONTAINING FLUID}

Let's investigate the joined conical-cylindrical-conical shells containing fluid in Fig. 1. $R_{1}$ is the radius of the cylinder, $R_{2}$ is the radius of the larger end of the cone. $L_{1}$ and $L_{2}$ are lengths of the cylinder and cone respectively. Conical shell theory using the Reissner-Mindlin assumption will be used to mobilize both conical and cylindrical shells.

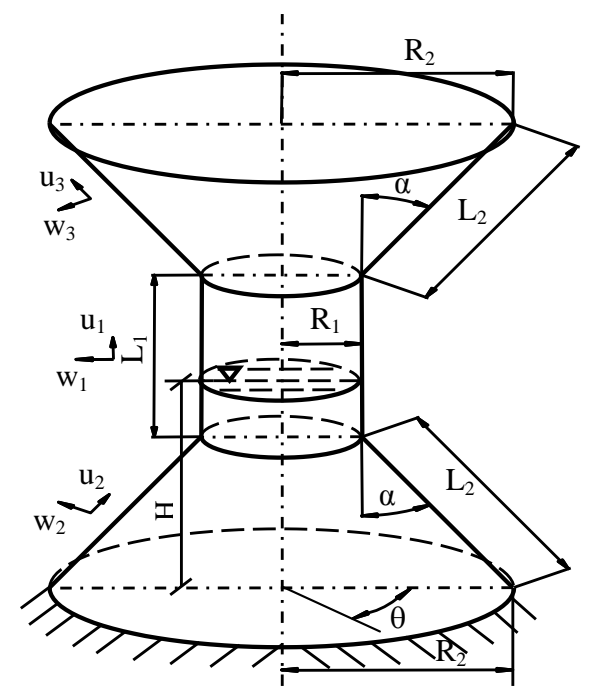

Fig. 1. Geometry of joined composite conical-cylindrical-conical shells containing fluid 


\subsection{Composite conical shell containing fluid formulation}

\subsubsection{Constitutive relations}

Consider a laminated composite shell of total thickness $h$ composed by $N$ orthotropic layers. The plane stress-reduced stiffness are calculated as

$$
\begin{aligned}
& Q_{11}=\frac{E_{1}}{1-v_{12} v_{21}}, \quad Q_{12}=\frac{v_{12} E_{2}}{1-v_{12} v_{21}}, \\
& Q_{22}=\frac{E_{2}}{1-v_{12} v_{21}}, \quad Q_{66}=G_{12}, \quad Q_{44}=G_{23}, \quad Q_{55}=G_{13}
\end{aligned}
$$

where $\mathrm{E}_{i}, \mathrm{G}_{i j}, v_{12}, v_{21}$ : elastic constants of the $k^{\text {th }}$ layer and the laminate stiffness coefficients $\left(A_{i j}, B_{i j}, D_{i j}, F_{i j}\right)$ are defined by

$$
\begin{aligned}
& A_{i j}=\sum_{k=1}^{N} \bar{Q}_{i j}^{k}\left(z_{k+1}-z_{k}\right), B_{i j}=\frac{1}{2} \sum_{k=1}^{N} \bar{Q}_{i j}^{k}\left(z_{k+1}^{2}-z_{k}^{2}\right), D_{i j}=\frac{1}{3} \sum_{k=1}^{N} \bar{Q}_{i j}^{k}\left(z_{k+1}^{3}-z_{k}^{3}\right)(i, j=1,2,6) \\
& F_{i j}=\sum_{k=1}^{N} Q_{i j}^{k}\left(z_{k+1}^{-} z_{k}\right)(i, j=4,5)
\end{aligned}
$$

where $z_{k-1}$ and $z_{k}$ are the boundaries of the $k^{\text {th }}$ layer.

\subsubsection{Strains, stress and internal forces resultant}

Following the Reissner-Mindlin assumption, the displacement components are assumed to be

$$
\begin{aligned}
& u(x, \theta, z, t)=u_{0}(x, \theta, t)+z \varphi_{x}(x, \theta, t), \quad v(x, \theta, z, t)=v_{0}(x, \theta, t)+z \varphi_{\theta}(x, \theta, t), \\
& w(x, \theta, z, t)=w_{0}(x, \theta, t)
\end{aligned}
$$

a) The strain-displacement relations of conical shell are (with $\left.R(x)=R_{1}+x \cdot \sin \alpha\right)$ :

$$
\begin{aligned}
& \varepsilon_{x}=\frac{\partial u_{0}}{\partial x}, k_{x}=\frac{\partial \varphi_{x}}{\partial x}, \varepsilon_{\theta}=\frac{1}{R}\left(u_{0} \sin \alpha+\frac{\partial v_{0}}{\partial \theta}+w_{0} \cos \alpha\right), k_{\theta}=\frac{1}{R}\left(\varphi_{x} \sin \alpha+\frac{\partial \varphi_{\theta}}{\partial \theta}\right), \\
& \varepsilon_{S \theta}=\frac{\partial v_{0}}{\partial s}+\frac{1}{R} \frac{\partial u_{0}}{\partial \theta}-\frac{\sin \alpha}{R} v_{0}, k_{x \theta}=\frac{1}{R} \frac{\partial \varphi_{x}}{\partial \theta}+\frac{\partial \varphi_{\theta}}{\partial x}-\frac{\sin \alpha}{R} \varphi_{\theta}, \gamma_{\theta Z}=\frac{-\cos \alpha}{R} v_{0}+\frac{1}{R} \frac{\partial w_{0}}{\partial \theta}+\varphi_{\theta}
\end{aligned}
$$

b) Force resultants-displacement relationships:

The force and moment resultants are expressed in terms of deformations for crossply axis-symmetric shell as follows

$$
\left[\begin{array}{l}
N \\
M \\
Q
\end{array}\right]=\left[\begin{array}{lll}
A & B & 0 \\
B & D & 0 \\
0 & 0 & F
\end{array}\right]\left[\begin{array}{l}
\varepsilon \\
k \\
\gamma
\end{array}\right]
$$


Substituting Eqs. (2) and (4) in Eqs. (5), the forces-displacements expressions for laminated composite conical shell are written as follows

$$
\begin{aligned}
& N_{x}=A_{11} \frac{\partial u_{0}}{\partial x}+\frac{A_{12}}{R}\left(u_{0} \sin \alpha+\frac{\partial v_{0}}{\partial \theta}+w_{0} \cos \alpha\right)+B_{11} \frac{\partial \varphi_{x}}{\partial x}+\frac{B_{12}}{R}\left(\varphi_{x} \sin \alpha+\frac{\partial \varphi_{\theta}}{\partial \theta}\right), \\
& N_{\theta}=A_{12} \frac{\partial u_{0}}{\partial x}+\frac{A_{22}}{R}\left(u_{0} \sin \alpha+\frac{\partial v_{0}}{\partial \theta}+w_{0} \cos \alpha\right)+B_{12} \frac{\partial \varphi_{x}}{\partial x}+\frac{B_{22}}{R}\left(\varphi_{x} \sin \alpha+\frac{\partial \varphi_{\theta}}{\partial \theta}\right), \\
& N_{x \theta}=A_{66}\left(\frac{\partial v_{0}}{\partial x}+\frac{1}{R} \frac{\partial u_{0}}{\partial \theta}-\frac{\sin \alpha}{R} v_{0}\right)+B_{66}\left(\frac{1}{R} \frac{\partial \varphi_{x}}{\partial \theta}+\frac{\partial \varphi_{\theta}}{\partial x}-\frac{\sin \alpha}{R} \varphi_{\theta}\right), \\
& M_{x}=B_{11} \frac{\partial u_{0}}{\partial x}+\frac{B_{12}}{R}\left(u_{0} \sin \alpha+\frac{\partial v_{0}}{\partial \theta}+\frac{w_{0} \cos \alpha}{R}\right)+D_{11} \frac{\partial \varphi_{x}}{\partial x}+\frac{D_{12}}{R}\left(\varphi_{x} \sin \alpha+\frac{\partial \varphi_{\theta}}{\partial \theta}\right), \\
& M_{\theta}=B_{12} \frac{\partial u_{0}}{\partial x}+\frac{B_{22}}{R}\left(u_{0} \sin \alpha+\frac{\partial v_{0}}{\partial \theta}+w_{0} \cos \alpha\right)+D_{12} \frac{\partial \varphi_{x}}{\partial x}+\frac{D_{22}}{R}\left(\varphi_{x} \sin \alpha+\frac{\partial \varphi_{\theta}}{\partial \theta}\right), \\
& M_{x \theta}=B_{66}\left(\frac{\partial v_{0}}{\partial x}+\frac{\partial u_{0}}{R \partial \theta}-\frac{\sin \alpha}{R} v_{0}\right)+D_{66}\left(\frac{1}{R} \frac{\partial \varphi_{x}}{\partial \theta}+\frac{\partial \varphi_{\theta}}{\partial x}-\frac{\sin \alpha}{R} \varphi_{\theta}\right), \\
& Q_{x}=k F_{55}\left(\frac{\partial w_{0}}{\partial x}+\varphi_{x}\right), \quad Q_{\theta}=k F_{44}\left(\frac{-\cos \alpha}{R} v_{0}+\frac{1}{R} \frac{\partial w_{0}}{\partial \theta}+\varphi_{\theta}\right),
\end{aligned}
$$

where $k$ is the shear correction factor $(k=5 / 6)$

\subsubsection{Equations of motion}

The equations of motion using the FSDT for laminated composite conical shell containing fluid are

$$
\begin{aligned}
& \frac{\partial N_{x}}{\partial x}+\frac{\sin \alpha}{R}\left(N_{x}-N_{\theta}\right)+\frac{1}{R} \frac{\partial N_{x \theta}}{\partial \theta}=I_{0} \ddot{u}_{0}+I_{1} \ddot{\varphi}_{x}, \\
& \frac{\partial N_{x \theta}}{\partial x}+\frac{2 \sin \alpha}{R} N_{x \theta}+\frac{1}{R} \frac{\partial N_{\theta}}{\partial \theta}+\frac{\cos \alpha}{R} Q_{\theta}=I_{0} \ddot{v}_{0}+I_{1} \ddot{\varphi}_{\theta}, \\
& \frac{\partial M_{x}}{\partial x}+\frac{\sin \alpha}{R}\left(M_{x}-M_{\theta}\right)+\frac{1}{R} \frac{\partial M_{x \theta}}{\partial \theta}-Q_{x}=I_{1} \ddot{u}_{0}+I_{2} \ddot{\varphi}_{x}, \\
& \frac{\partial M_{x \theta}}{\partial x}+\frac{2 \sin \alpha}{R} M_{x \theta}+\frac{1}{R} \frac{\partial M_{\theta}}{\partial \theta}-Q_{\theta}=I_{1} \ddot{v}_{0}+I_{2} \ddot{\varphi}_{\theta}, \\
& \frac{\partial Q_{x}}{\partial x}+\frac{1}{R} \frac{\partial Q_{\theta}}{\partial \theta}+\frac{\sin \alpha}{R} Q_{x}-\frac{\cos \alpha}{R} N_{\theta}-P \cos \alpha=I_{0} \ddot{w}_{0},
\end{aligned}
$$

where $u_{0}, v_{0}, w_{0}$ : displacement, $\varphi_{x}, \varphi_{\theta}$ : rotation of tangents along the $x$ and $\theta$. And $I_{i}=\sum_{k=1}^{N} \int_{z_{k}}^{z_{k+1}} \rho^{(k)} z^{i} d z,(i=0,1,2)$, where $\rho^{(k)}$ is the material mass density of the $k^{\text {th }}$ layer.

Substituting $\alpha=0$ in Eqs. (6) and (7), the forces-displacements relations and the equations of motion for laminated composite cylindrical shell containing fluid can be obtained [27]. 


\subsubsection{Fluid equations}

The potential function $\Phi(r, \theta, x, t)$ satisfies the Laplace equation in cylindrical coordinates $(r, \theta, x)$

$$
\frac{\partial^{2} \Phi}{\partial r^{2}}+\frac{1}{r} \frac{\partial \Phi}{\partial r}+\frac{1}{r^{2}} \frac{\partial^{2} \Phi}{\partial \theta^{2}}+\frac{\partial^{2} \Phi}{\partial x^{2}}=0
$$

Then, the Bernoulli equation is written by

$$
\frac{\partial \Phi}{\partial t}+\frac{P}{\rho_{f}}=0
$$

By linearizing this expression, the pressures on the internal regions are

$$
P=-\left.\rho \frac{\partial \Phi}{\partial t}\right|_{\Sigma}
$$

where $\Sigma$ is the portion of the structure's surface in contact with fluid.

The condition of impermeability of the surface of shell in contact with fluid can be expressed as

$$
v_{f}=\left.\frac{\partial \Phi}{\partial r}\right|_{\Sigma}=\left.\frac{\partial w_{0}}{\partial t}\right|_{\Sigma}
$$

where $w_{0}$ is the normal displacement of the shell, $v_{f}$ is the velocity of fluid.

The hydrodynamic pressure acting on the cylindrical shell is then defined by [27]

$$
P=-\rho_{f} \frac{1}{m+k_{n} R I_{m+1}\left(k_{n} R\right) / I_{m}\left(k_{n} R\right)} \frac{\partial^{2} w_{0}}{\partial t^{2}}=m^{*} \frac{\partial^{2} w_{0}}{\partial t^{2}} .
$$

This value will be introduced in (7) in order to establish the Dynamic Stiffness Matrix for the studied structure.

\subsubsection{Continuity conditions}

The continuity conditions at the conical-cylindrical shell joint can be obtained from Caresta and Kessissoglou [19] as follows

$$
\begin{aligned}
& u_{1}=u_{2} \cos \alpha-w_{2} \sin \alpha, \quad v_{1}=v_{2}, \quad w_{1}=u_{2} \sin \alpha+w_{2} \cos \alpha, \quad \frac{\partial w_{1}}{\partial x_{1}}=\frac{\partial w_{2}}{\partial x_{2}}, \\
& N_{x 1}=N_{x 2} \cos \alpha-Q_{x 2} \sin \alpha, Q_{x 1}=N_{x 2} \sin \alpha+Q_{x 2} \cos \alpha, M_{x \theta 1}=M_{x \theta 2}, M_{x 1}=M_{x 2} .
\end{aligned}
$$

\section{CONTINUOUS ELEMENT FORMULATION FOR THICK LAMINATED COMPOSITE JOINED CONICAL-CYLINDRICAL-CONICAL SHELLS CONTAINING FLUID}

\subsection{Strong formulation}

Here, the same state-vector $\mathbf{y}=\left\{u_{0}, v_{0}, w_{0}, \varphi_{x}, \varphi_{\theta}, N_{x}, N_{x \theta}, Q_{x}, M_{x}, M_{x \theta}\right\}^{T}$. Next, the Lévy series expansion for state variables is written as 


$$
\begin{aligned}
& \left\{u_{0}(x, \theta, t), w_{0}(x, \theta, t), \varphi_{\theta}(x, \theta, t), N_{x}(x, \theta, t), Q_{x}(x, \theta, t), M_{x}(x, \theta, t)\right\}^{T}= \\
& =\sum_{m=1}^{\infty}\left\{u_{m}(x), w_{m}(x), \varphi_{\theta_{m}}(x), N_{x_{m}}(x), Q_{x_{m}}(x), M_{x_{m}}(x)\right\}^{T} \cos m \theta e^{i \omega t}, \\
& \left\{v_{0}(x, \theta, t), \varphi_{x}(x, \theta, t), N_{x \theta}(x, \theta, t), M_{x \theta}(x, \theta, t)\right\}^{T}= \\
& =\sum_{m=1}^{\infty}\left\{v_{m}(x), \varphi_{x_{m}}(x), N_{x \theta_{m}}(x), M_{x \theta_{m}}(x)\right\}^{T} \sin m \theta e^{i \omega t},
\end{aligned}
$$

where $m$ is the number of circumferential wave.

Substituting (14) in Eqs. (6) and (7), a system of ordinary differential equations in the $x$-coordinate for the $m^{\text {th }}$ mode can be expressed in the matrix form for each circumferential mode $m$ as [25-28]

$$
\begin{aligned}
& \frac{d u_{m}}{d x}=c_{4} \sin \alpha \cdot u_{m}+m c_{4} v_{m}+c_{4} \cos \alpha . w_{m}+c_{5} \sin \alpha \cdot \varphi_{x m}+m c_{5} \varphi_{\theta m}+\frac{D_{11}}{c_{1}} N_{x m}-\frac{B_{11}}{c_{1}} M_{x m} ， \\
& \frac{d v_{m}}{d x}=\frac{m}{R} u_{m}-\frac{\sin \alpha}{R} v_{m}-\frac{D_{66}}{c_{10}} N_{x \theta m}+\frac{B_{66}}{c_{10}} M_{x \theta m}, \quad \frac{d w_{m}}{d x}=-\varphi_{x m}+\frac{1}{k F_{55}} Q_{x m} ， \\
& \frac{d \varphi_{x m}}{d x}=c_{2} \sin \alpha \cdot u_{m}+m c_{2} v_{m}+c_{2} \cos \alpha \cdot w_{m}+c_{3} \sin \alpha \cdot \varphi_{x m}+m c_{3} \varphi_{\theta m}-\frac{B_{11}}{c_{1}} N_{x m}+\frac{A_{11}}{c_{1}} M_{x m} \text { ， } \\
& \frac{d \varphi_{\theta m}}{d x}=\frac{m}{R} \varphi_{x m}-\frac{\sin \alpha}{R} \varphi_{\theta m}+\frac{B_{66}}{c_{10}} N_{x \theta m}-\frac{A_{66}}{c_{10}} M_{x \theta m} \\
& \frac{d N_{x m}}{d x}=\left(c_{6} \sin ^{2} \alpha-I_{0} \omega^{2}\right) u_{m}+m c_{6} \sin \alpha . v_{m}+c_{6} \sin \alpha \cos \alpha . w_{m}+\left(c_{7} \sin ^{2} \alpha-I_{1} \omega^{2}\right) \varphi_{x m}+ \\
& +m c_{7} \sin \alpha \cdot \varphi_{\theta m}-\sin \alpha\left(c_{4}+\frac{1}{R}\right) N_{x m}-\frac{m}{R} N_{x \theta m}-c_{2} \sin \alpha \cdot M_{x m}, \\
& \frac{d N_{x \theta m}}{d x}=m c_{6} \sin \alpha \cdot u_{m}+\left(m^{2} c_{6}+\frac{k F_{44} \cos ^{2} \alpha}{R^{2}}-I_{0} \omega^{2}\right) v_{m}+m \cos \alpha\left(c_{6}+\frac{k F_{44}}{R^{2}}\right) w_{m}+ \\
& +m c_{7} \sin \alpha \cdot \varphi_{x m}+\left(m^{2} c_{7}-\frac{k F_{44} \cos \alpha}{R}-I_{1} \omega^{2}\right) \varphi_{\theta m}-m c_{4} N_{x m}-\frac{2 \sin \alpha}{R} N_{x \theta m}-m c_{2} M_{x m} \text {, } \\
& \frac{d Q_{x m}}{d x}=c_{6} \sin \alpha \cos \alpha \cdot u_{m}+m \cos \alpha\left(c_{6}+\frac{k F_{44}}{R^{2}}\right) v_{m}+\left(c_{6} \cos ^{2} \alpha+\frac{m^{2} k F_{44}}{R^{2}}-I_{0} \omega^{2}-m^{*} \omega^{2}\right) w_{m}+ \\
& +c_{7} \sin \alpha \cos \alpha \cdot \varphi_{x m}+\left(m c_{7} \cos \alpha-\frac{m k F_{44}}{R}\right) \varphi_{\theta m}-c_{4} \cos \alpha \cdot N_{x m}-\frac{\sin \alpha}{R} Q_{x m}-c_{2} \cos \alpha \cdot M_{x m}, \\
& \frac{d M_{x m}}{d x}=\left(2 c_{8} \sin ^{2} \alpha-I_{1} \omega^{2}\right) u_{m}+2 m c_{8} \sin \alpha . v_{m}+2 c_{8} \sin \alpha \cos \alpha . w_{m}+\left(2 c_{9} \sin ^{2} \alpha-I_{2} \omega^{2}\right) \varphi_{x m}+ \\
& +2 m c_{9} \sin \alpha \cdot \varphi_{\theta m}-2 c_{5} \sin \alpha \cdot N_{x m}+Q_{x}-\left[2 \sin \alpha\left(c_{3}+\frac{1}{R}\right)\right] M_{x m}-\frac{m}{R} M_{x \theta m}, \\
& \frac{d M_{x \theta m}}{d x}=m c_{8} \sin \alpha \cdot u_{m}+\left(m^{2} c_{8}-\frac{k F_{44} \cos \alpha}{R}-I_{1} \omega^{2}\right) v_{m}+m\left(c_{8} \cos \alpha-\frac{k F_{44}}{R}\right) w_{m}+ \\
& +m c_{9} \sin \alpha \cdot \varphi_{x m}+\left(m^{2} c_{9}+k F_{44}-I_{2} \omega^{2}\right) \varphi_{\theta m}-m c_{5} \cdot N_{x m}-m c_{3} M_{x m}-\frac{2 \sin \alpha}{R} \cdot M_{x \theta m},
\end{aligned}
$$


with

$$
\begin{aligned}
& c_{1}=A_{11} D_{11}-B_{11}^{2}, \quad c_{2}=\left(A_{12} B_{11}-A_{11} B_{12}\right) / R c_{1}, \quad c_{3}=\left(B_{11} B_{12}-A_{11} D_{12}\right) / R c_{1}, \\
& c_{4}=\left(B_{11} B_{12}-A_{12} D_{11}\right) / R c_{1}, \quad c_{5}=\left(B_{11} D_{12}-B_{12} D_{11}\right) / R c_{1}, \\
& c_{6}=\left(A_{12} c_{4}+B_{12} c_{2}+A_{22} / R\right) / R, \quad c_{7}=\left(A_{12} c_{5}+B_{12} c_{3}+B_{22} / R\right) / R, \\
& c_{8}=\left(B_{12} c_{4}+D_{12} c_{2}+B_{22} / R\right) / R, \quad c_{9}=\left(B_{12} c_{5}+D_{12} c_{3}+D_{22} / R\right) / R, \\
& c_{10}=B_{66}^{2}-A_{66} D_{66} .
\end{aligned}
$$

Eq. (15) can be expressed in the matrix form for each circumferential mode $m$

$$
\frac{d \mathbf{y}_{m}}{d x}=\mathbf{A}_{m} \mathbf{y}_{m}
$$

with $\mathbf{A}_{m}$ is a $10 \times 10$ matrix (see Appendix).

\subsection{Dynamic transfer matrix, dynamic stiffness matrix $K(\omega)$}

The dynamic transfer matrix $[\mathbf{T}]_{m}$ is given by: $T_{m}(\omega)=e^{\int_{0}^{L} A_{m}(x, \omega) d x}$.

Then $[\mathbf{T}]_{m}$ is separated into four blocks

$$
[\mathbf{T}]_{m}=\left[\begin{array}{ll}
\mathbf{T}_{11} & \mathbf{T}_{12} \\
\mathbf{T}_{21} & \mathbf{T}_{22}
\end{array}\right]
$$

Finally, the dynamic stiffness matrix $[K(\omega)]_{m}$ for conical shell containing fluid is determined by

$$
[\mathbf{K}(\omega)]_{m}=\left[\begin{array}{cc}
\mathbf{T}_{12}^{-1} \mathbf{T}_{11} & -\mathbf{T}_{12}^{-1} \\
\mathbf{T}_{21}-\mathbf{T}_{22} \mathbf{T}_{12}^{-1} \mathbf{T}_{11} & \mathbf{T}_{22} \mathbf{T}_{12}^{-1}
\end{array}\right]_{m}
$$

Similarly, we can obtain the dynamic stiffness matrix $[\mathbf{K}(\omega)]_{m}$ for cylindrical shell containing fluid [27].

The assembly procedure of the finite element method is used to construct the Dynamic Stiffness Matrix for combined conical-cylindrical-conical shells containing fluid.

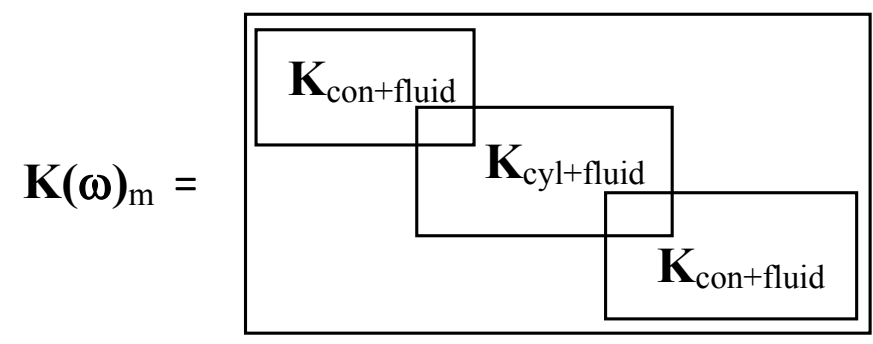

Natural frequencies will be extracted from the harmonic responses of the structure by using the procedure detailed in [25-28]. 


\section{NUMERICAL RESULTS AND DISCUSSION}

\subsection{Modal analysis}

A computer program based on Matlab is developed using DSM to solve a number of numerical examples on free vibration of composite joined conical-cylindrical-conical shells containing fluid with different fluid level and geometries shell. Fundamental frequencies will be validated with respect to those of literature and to the results obtained by FEM.

First, natural frequencies are validated for a Free-clamped cross-ply laminated composite cylindrical shells containing fluid having the following dimensions and material: $E_{1}=206.9 \mathrm{GPa} ; E_{2}=18.62 \mathrm{GPa} ; v_{12}=0.28, G_{12}=4.48 \mathrm{GPa} ; G_{13}=4.48 \mathrm{GPa}$; $G_{23}=2.24 \mathrm{GPa} ; \rho=2048 \mathrm{~kg} / \mathrm{m}^{3}$, layers $\left[0^{0} / 90^{0} / 0^{0} / 90^{0}\right] ; h=9.525 \mathrm{~mm} ; R=0.1905 \mathrm{~m}$; $L=0.381 \mathrm{~m}, \rho_{f}=1000 \mathrm{~kg} / \mathrm{m}^{3}$. Our results comparing to FEM-ANSYS and Xi et al. values [2] are illustrated in Tab. 1.

Table 1. Comparison of natural frequencies of laminated composite cylindrical shells partially filled with fluid by FEM and by CEM in comparison with results of Xi et al. [2]

\begin{tabular}{|c|c|c|c|c|c|c|}
\hline $\begin{array}{c}\text { Fluid } \\
\text { levers }\end{array}$ & $\begin{array}{c}\text { Mode } \\
(n, m)\end{array}$ & $\begin{array}{c}\mathrm{Xi}[2] \\
(1)\end{array}$ & $\begin{array}{c}\text { FEM } \\
(60 \times 19 \times 10)\end{array}$ & $\begin{array}{c}\text { CEM } \\
(2)\end{array}$ & $\begin{array}{c}\text { Error }(\%) \\
(1)-(2)\end{array}$ & $\begin{array}{c}\text { \% Reduction } \\
\text { with } H / L=0\end{array}$ \\
\hline \multirow{3}{*}{$H / L=0$} & $1(1,2)$ & 419,54 & 454,15 & 460,3 & 1,35 & - \\
\cline { 2 - 7 } & $2(1,3)$ & 517,2 & 561,02 & 566,2 & 0,92 & - \\
\cline { 2 - 7 } & $3(1,1)$ & 645,24 & 677,56 & 679,12 & 0,23 & - \\
\hline \multirow{3}{*}{$H / L=0.5$} & $1(1,2)$ & 377,2 & 401,12 & 402,52 & 0,35 & 12,55 \\
\cline { 2 - 7 } & $2(1,3)$ & 440,64 & 483,49 & 482,42 & $-0,22$ & 14,80 \\
\cline { 2 - 7 } & $3(1,1)$ & 581,98 & 606,03 & 614,61 & 1,42 & 9,50 \\
\hline \multirow{3}{*}{$H / L=1$} & $1(1,2)$ & 206,41 & 236,06 & 235,6 & $-0,19$ & 48,80 \\
\cline { 2 - 7 } & $2(1,3)$ & 280,09 & 303,28 & 297,7 & $-1,84$ & 47,42 \\
\cline { 2 - 7 } & $3(1,1)$ & 314,85 & 355,67 & 372,36 & 4,69 & 45,17 \\
\hline
\end{tabular}

The next step of our research on composite axis-symmetric joined shell is obviously to validate the presented formulation for this type of structure. Consider the freeclamped (F-C) joined cross-ply laminated conical-cylindrical shells having the following dimensions and material: $L / R_{1}=1 ; h / R_{1}=0.01 ; h=2 \mathrm{~mm} ; L_{i}=L, E_{1}=135 \mathrm{Gpa}$; $\alpha=0^{0}, 30^{0}, 60^{0} ; E_{2}=8.8 \mathrm{Gpa} ; \mathrm{G}_{12}=4.47 \mathrm{Gpa} ; v_{12}=0.33 ; \rho=1600 \mathrm{~kg} / \mathrm{m}^{3}$. Our results comparing to FEM-ANSYS values with different meshes and with analytical solutions of Kouchakzadeh [20] are illustrated in Tab. 2.

The agreement between FEM, CEM and $\mathrm{Xi}$ results, Kouchakzadeh results is very good in Tab. 1 and Tab 2. 
Table 2. The lowest fundamental frequency parameter $\Omega_{1}=\omega R_{1}\left[\rho h / A_{11}\right]^{1 / 2}$ and corresponding circumferential wave numbers $(m)$ for various types lamination sequences of joined cross-ply laminated conical-cylindrical shells (FC boundary condition)

\begin{tabular}{|c|c|c|c|c|c|c|c|}
\hline No & $\begin{array}{l}\text { Cone } \\
\text { angles }\end{array}$ & Layers & $\begin{array}{c}\text { Kouchakzadeh [20] } \\
\text { (1) }\end{array}$ & $\begin{array}{l}\text { Ansys } \\
40 \times 10\end{array}$ & $\begin{array}{l}\text { Ansys } \\
80 \times 20\end{array}$ & $\begin{array}{l}\text { CEM } \\
(2)\end{array}$ & $\begin{array}{c}\text { Errors (\%) } \\
\quad(2)-(1)\end{array}$ \\
\hline 1 & \multirow{12}{*}{$\alpha=0^{0}$} & {$[0 / 90]$} & $0.0455(4)$ & 0.0454 & 0.0454 & 0.0458 & 0.66 \\
\hline 2 & & {$[90 / 0]$} & $0.0455(4)$ & 0.0453 & 0.0453 & 0.0457 & 0.44 \\
\hline 3 & & {$[0 / 0 / 0]$} & $0.0288(5)$ & 0.0286 & 0.0286 & 0.0289 & 0.35 \\
\hline 4 & & {$[0 / 90 / 0]$} & $0.0365(5)$ & 0.0362 & 0.0362 & 0.0366 & 0.27 \\
\hline 5 & & {$[0 / 0 / 90]$} & $0.0403(4)$ & 0.0401 & 0.0401 & 0.0405 & 0.49 \\
\hline 6 & & {$[0 / 90 / 90]$} & $0.0567(4)$ & 0.0565 & 0.0565 & 0.0570 & 0.53 \\
\hline 7 & & {$[90 / 90 / 0]$} & $0.0577(4)$ & 0.0576 & 0.0576 & 0.0578 & 0.17 \\
\hline 8 & & {$[90 / 90 / 90]$} & $0.1183(3)$ & 0.1187 & 0.1188 & 0.1183 & 0.00 \\
\hline 9 & & {$[0 / 90]_{2}$} & $0.0533(4)$ & 0.0531 & 0.0532 & 0.0535 & 0.37 \\
\hline 10 & & {$[0 / 90]_{S}$} & $0.0442(4)$ & 0.0438 & 0.0440 & 0.0443 & 0.23 \\
\hline 11 & & {$[90 / 0]_{2}$} & $0.0533(4)$ & 0.0531 & 0.0532 & 0.0535 & 0.37 \\
\hline 12 & & {$[90 / 0]_{S}$} & $0.0593(3)$ & 0.0591 & 0.0592 & 0.0595 & 0.34 \\
\hline 1 & \multirow{12}{*}{$\alpha=30^{0}$} & [0/90] & $0.0366(4)$ & 0.0363 & 0.0363 & 0.0367 & 0.27 \\
\hline 2 & & {$[90 / 0]$} & $0.0367(4)$ & 0.0364 & 0.0364 & 0.0367 & 0.00 \\
\hline 3 & & {$[0 / 0 / 0]$} & $0.0238(4)$ & 0.0236 & 0.0236 & 0.0239 & 0.42 \\
\hline 4 & & {$[0 / 90 / 0]$} & $0.0309(4)$ & 0.0306 & 0.0306 & 0.0310 & 0.32 \\
\hline 5 & & {$[0 / 0 / 90]$} & $0.0313(4)$ & 0.0310 & 0.0310 & 0.0313 & 0.00 \\
\hline 6 & & [0/90/90] & $0.0492(4)$ & 0.0489 & 0.0489 & 0.0494 & 0.40 \\
\hline 7 & & {$[90 / 90 / 0]$} & $0.0500(4)$ & 0.0498 & 0.0498 & 0.0502 & 0.40 \\
\hline 8 & & [90/90/90] & $0.1389(3)$ & 0.1394 & 0.1395 & 0.1392 & 0.22 \\
\hline 9 & & {$[0 / 90]_{2}$} & $0.0468(4)$ & 0.0466 & 0.0466 & 0.0470 & 0.43 \\
\hline 10 & & {$[0 / 90]_{S}$} & $0.0384(4)$ & 0.0381 & 0.0381 & 0.0385 & 0.26 \\
\hline 11 & & {$[90 / 0]_{2}$} & $0.0469(4)$ & 0.0467 & 0.0467 & 0.0471 & 0.42 \\
\hline 12 & & {$[90 / 0]_{S}$} & $0.0530(3)$ & 0.0528 & 0.0528 & 0.0532 & 0.38 \\
\hline 1 & \multirow{12}{*}{$\alpha=60^{0}$} & {$[0 / 90]$} & $0.0250(3)$ & 0.0248 & 0.0248 & 0.0253 & 1.19 \\
\hline 2 & & [90/0] & $0.0249(3)$ & 0.0247 & 0.0247 & 0.0252 & 1.19 \\
\hline 3 & & {$[0 / 0 / 0]$} & $0.0181(4)$ & 0.0178 & 0.0178 & 0.0182 & 0.55 \\
\hline 4 & & {$[0 / 90 / 0]$} & $0.0241(4)$ & 0.0238 & 0.0238 & 0.0243 & 0.82 \\
\hline 5 & & {$[0 / 0 / 90]$} & $0.0223(3)$ & 0.0222 & 0.0222 & 0.0226 & 1.33 \\
\hline 6 & & [0/90/90] & $0.0315(3)$ & 0.0313 & 0.0313 & 0.0318 & 0.94 \\
\hline 7 & & {$[90 / 90 / 0]$} & $0.0320(3)$ & 0.0318 & 0.0318 & 0.0323 & 0.93 \\
\hline 8 & & [90/90/90] & $0.0963(3)$ & 0.0967 & 0.0968 & 0.0979 & 1.63 \\
\hline 9 & & {$[0 / 90]_{2}$} & $0.0302(3)$ & 0.0300 & 0.0300 & 0.0305 & 0.98 \\
\hline 10 & & {$[0 / 90]_{S}$} & $0.0285(3)$ & 0.0281 & 0.0282 & 0.0287 & 0.70 \\
\hline 11 & & {$[90 / 0]_{2}$} & $0.0302(3)$ & 0.0300 & 0.0300 & 0.0304 & 0.66 \\
\hline 12 & & {$[90 / 0]_{S}$} & $0.0342(3)$ & 0.0348 & 0.0248 & 0.0344 & 0.58 \\
\hline
\end{tabular}


Table 3. The fundamental frequency $\omega(\mathrm{Hz})$ corresponding to circumferential wave numbers $(\mathrm{m})$ for various fluid level of joined cross-ply laminated conical-cylindrical-conical shells containing fluid, $n=1$

\begin{tabular}{|c|c|c|c|c|c|}
\hline $\begin{array}{l}\text { Fluid } \\
\text { Level }\end{array}$ & $m$ & $\begin{array}{c}\text { Ansys } \\
60 \times 19 \times 10 \\
(1)\end{array}$ & $\begin{array}{c}\text { CEM } \\
(2)\end{array}$ & $\begin{array}{c}\text { Errors }(\%) \\
(2)-(1)\end{array}$ & $\begin{array}{l}\% \text { Reduction } \\
\text { with } H / L=0\end{array}$ \\
\hline \multirow{5}{*}{$H=0$} & 1 & 85.47 & 85.70 & 0.27 & - \\
\hline & 2 & 28.59 & 28.70 & 0.38 & - \\
\hline & 3 & 23.27 & 23.40 & 0.56 & - \\
\hline & 4 & 41.58 & 41.70 & 0.29 & - \\
\hline & 5 & 67.05 & 67.20 & 0.22 & - \\
\hline \multirow{5}{*}{$H=0.5 H_{1}$} & 1 & 80.23 & 80.80 & 0.70 & 5.72 \\
\hline & 2 & 28.00 & 28.00 & 0 & 2.44 \\
\hline & 3 & 23.12 & 23.20 & 0.34 & 0.85 \\
\hline & 4 & 41.72 & 41.60 & 0.29 & 0.24 \\
\hline & 5 & 65.18 & 65.00 & 0.28 & 3.27 \\
\hline \multirow{5}{*}{$H=H_{1}$} & 1 & 75.25 & 75.50 & 0.33 & 11.9 \\
\hline & 2 & 27.36 & 27.30 & 0.22 & 4.88 \\
\hline & 3 & 23.00 & 23.00 & 0 & 1.71 \\
\hline & 4 & 41.30 & 41.40 & 0.24 & 0.72 \\
\hline & 5 & 56.18 & 56.30 & 0.21 & 16.22 \\
\hline \multirow{5}{*}{$H=H_{1}+0.5 H_{2}$} & 1 & 28.67 & 28.90 & 0.80 & 66.28 \\
\hline & 2 & 9.79 & 10.30 & 4.95 & 64.11 \\
\hline & 3 & 9.95 & 10.30 & 3.40 & 55.98 \\
\hline & 4 & 18.25 & 18.30 & 0.27 & 56.12 \\
\hline & 5 & 21.36 & 21.20 & 0.75 & 68.45 \\
\hline \multirow{5}{*}{$H=H_{1}+H_{2}$} & 1 & 21.66 & 22.20 & 2.43 & 74.10 \\
\hline & 2 & 9.47 & 8.80 & 7.61 & 69.34 \\
\hline & 3 & 10.18 & 9.40 & 8.30 & 59.89 \\
\hline & 4 & 17.92 & 17.10 & 4.80 & 58.99 \\
\hline & 5 & 21.22 & 20.20 & 5.05 & 69.94 \\
\hline \multirow{5}{*}{$H=H_{1}+H_{2}+0.5 H_{3}$} & 1 & 20.10 & 20.10 & 0 & 76.55 \\
\hline & 2 & 6.60 & 6.80 & 2.94 & 76.31 \\
\hline & 3 & 5.71 & 6.00 & 4.83 & 74.36 \\
\hline & 4 & 11.10 & 11.40 & 2.63 & 72.66 \\
\hline & 5 & 18.97 & 18.70 & 1.44 & 72.17 \\
\hline \multirow{5}{*}{$H=H_{1}+H_{2}+H_{3}$ (full fluid) } & 1 & 18.46 & 18.40 & 0.33 & 78.53 \\
\hline & 2 & 5.52 & 5.90 & 6.44 & 79.44 \\
\hline & 3 & 5.23 & 5.20 & 0.58 & 77.78 \\
\hline & 4 & 10.61 & 10.10 & 5.05 & 75.78 \\
\hline & 5 & 18.60 & 17.40 & 6.90 & 74.11 \\
\hline
\end{tabular}




\subsection{Effects of fluid level}

Natural frequencies are computed for a Free-clamped joined cross-ply laminated composite conical-cylindrical-conical shells containing fluid having the following dimensions and material: $L_{1} / R_{1}=2 ; R_{1}=0.285 ; h=2 \mathrm{~mm} ; L_{1}=2 L_{2}, E_{1}=10.58 \mathrm{GPa}$; $\alpha=9^{0} ; E_{2}=2.64 \mathrm{GPa} ; G_{13}=G_{12}=1.02 \mathrm{GPa} ; v_{12}=0.17 ; \rho=1600 \mathrm{~kg} / \mathrm{m}^{3}, \rho_{f}=1000$ $\mathrm{kg} / \mathrm{m}^{3}$, layers $\left[0^{0} / 90^{0} / 0^{0} / 90^{0}\right]$. Our results comparing to FEM values are illustrated in Tab. 3.

CEM results give a good agreement with FEM solutions. The exactness of FEM depends on the choice of mesh but three continuous elements of CEM are enough to give exact results for all range of frequencies including medium and high frequencies. It is seen from Tab. 3 that the present model for joined cross-ply laminated conical-cylindricalconical shells containing fluid with free-clamped boundary conditions is validated. Errors when comparing with results of FEM varies from 0 to 8.30 percents which are very small and therefore the accuracy of our developed continuous element is confirmed.

Fig. 2 illustrates the effects of fluid level on fundamental frequencies of free-clamped laminated composite combined conical-cylindrical-conical shells containing fluid.
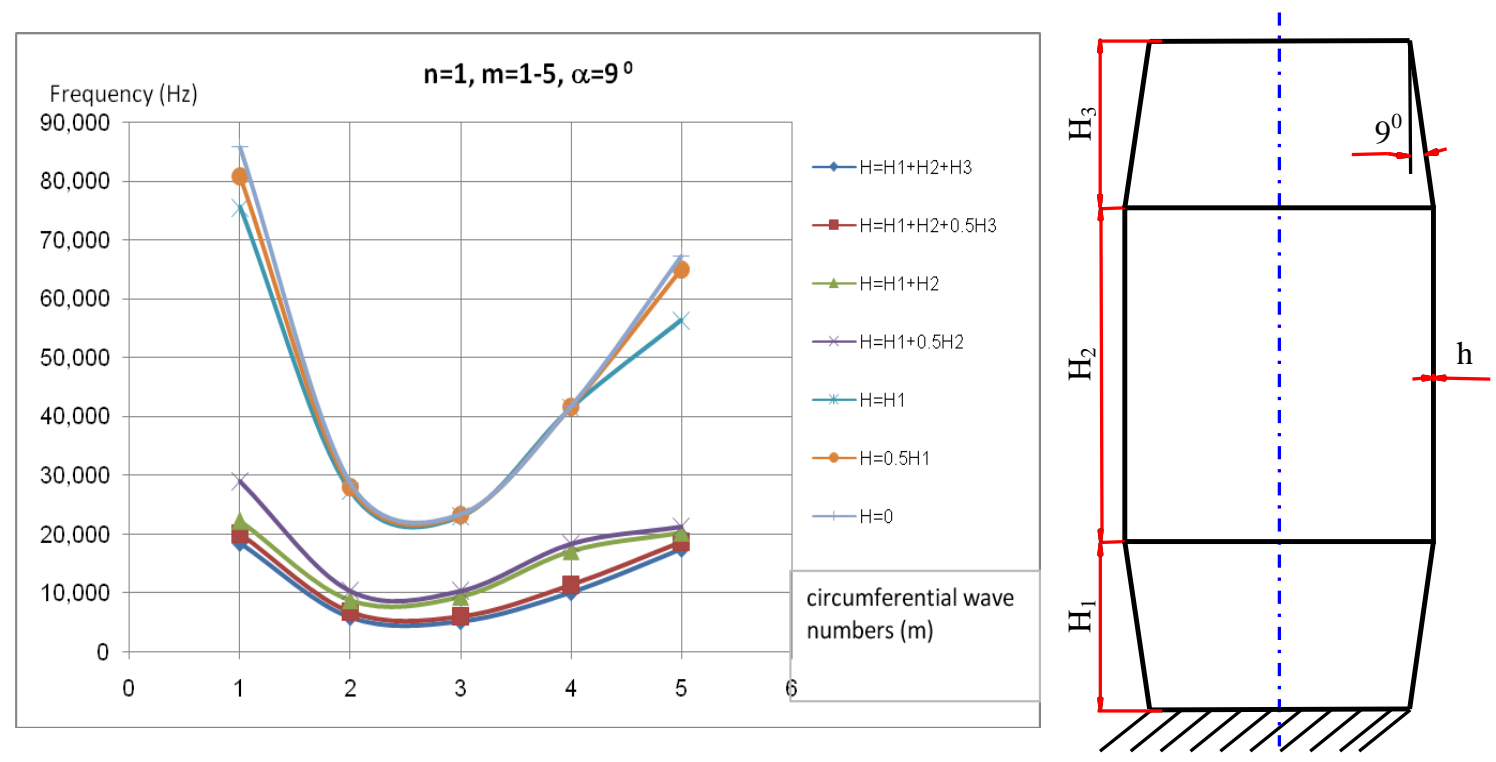

Fig. 2. Effect of fluid level on fundamental frequencies of combined conical-cylindrical-conical shells containing fluid

From the figure it can be seen clearly that the reduction of the natural frequency of composite combined conical-cylindrical-conical shells containing fluid as fluid level increases. Filled fluid can reduce significantly the natural frequency of a laminated composite conical-cylindrical-conical shells fill-fluid. From the Tab. 3 it can be seen clearly that the reduction of the natural frequency of the shell decreases first slowly then quickly as the fluid height increases. 
Table 4. The fundamental frequency $\omega(\mathrm{Hz})$ corresponding to circumferential wave numbers $(m)$ for various ratio $L_{1} / R_{1}$ of joined cross-ply laminated conical-cylindrical-conical shells containing fluid, $n=1$

\begin{tabular}{|c|c|c|c|c|}
\hline Ratio $L_{1} / R_{1}$ & $\begin{array}{l}\text { Fluid } \\
\text { Level }\end{array}$ & $m$ & CEM & $\begin{array}{c}\text { \% Reduction } \\
\text { with } H=0\end{array}$ \\
\hline \multirow{10}{*}{1} & \multirow{5}{*}{$H=0$} & 1 & 210.1 & - \\
\hline & & 2 & 94.8 & - \\
\hline & & 3 & 47.0 & - \\
\hline & & 4 & 43.5 & - \\
\hline & & 5 & 63.2 & - \\
\hline & \multirow{5}{*}{ full fluid } & 1 & 72.2 & 65.64 \\
\hline & & 2 & 27.3 & 71.20 \\
\hline & & 3 & 13.1 & 72.13 \\
\hline & & 4 & 12.4 & 71.49 \\
\hline & & 5 & 18.5 & 70.73 \\
\hline \multirow{10}{*}{2} & \multirow{5}{*}{$H=0$} & 1 & 85.7 & - \\
\hline & & 2 & 28.7 & - \\
\hline & & 3 & 23.4 & - \\
\hline & & 4 & 41.7 & - \\
\hline & & 5 & 67.2 & - \\
\hline & \multirow{5}{*}{ full fluid } & 1 & 21.6 & 74.80 \\
\hline & & 2 & 6.3 & 78.05 \\
\hline & & 3 & 5.3 & 77.35 \\
\hline & & 4 & 10.2 & 75.54 \\
\hline & & 5 & 17.6 & 73.81 \\
\hline \multirow{10}{*}{6} & \multirow{5}{*}{$H=0$} & 1 & 11.3 & - \\
\hline & & 2 & 12.5 & - \\
\hline & & 3 & 26.8 & - \\
\hline & & 4 & 35.5 & - \\
\hline & & 5 & 53.4 & - \\
\hline & \multirow{5}{*}{ full fluid } & 1 & 2.0 & 82.30 \\
\hline & & 2 & 2.3 & 81.60 \\
\hline & & 3 & 5.2 & 80.60 \\
\hline & & 4 & 7.6 & 78.59 \\
\hline & & 5 & 12.6 & 76.40 \\
\hline
\end{tabular}




\subsection{Effects of shell geometries}

Natural frequencies are computed for a Free-clamped joined cross-ply laminated composite conical-cylindrical-conical shells containing fluid with ratio $L_{1} / R_{1}=1 ; 2 ; 6$ $\left(R_{1}=0.285\right)$. Results are illustrated in Tab. 4 .

Fig. 3 illustrates the effects of shell geometries on fundamental frequencies of freeclamped laminated composite combined conical-cylindrical-conical shells containing fluid.

From the figure it can be seen clearly that natural frequency of composite joined cross-ply laminated conical-cylindrical-conical shells containing fluid decreases as ratio $L_{1} / R_{1}$ increases. The natural frequencies of this laminated shells decrease significantly as mode $m=1$ or 2 and $n=1$.
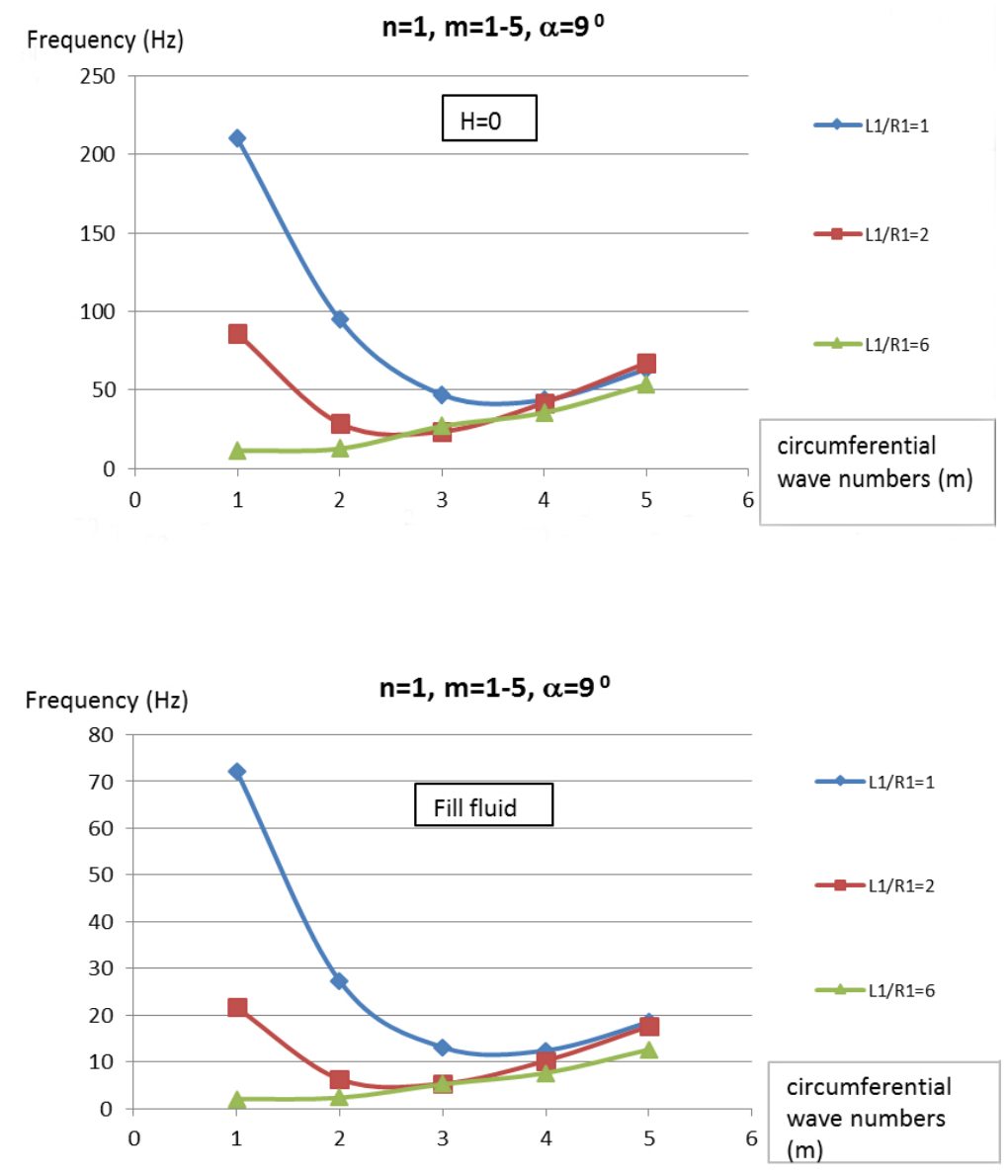

Fig. 3. Effect of shell geometries on fundamental frequencies of combined conical-cylindrical-conical shells containing fluid 


\section{CONCLUSIONS}

Based on the numerical results presented in this paper, the following conclusions may be drawn:

+ Various test cases confirm that Continuous Element Method allows computing the natural frequencies of thick joined cross-ply laminated conical-cylindrical-conical shells containing fluid with high accuracy for any frequency range. Using minimum meshing for complex structures, this model accelerates the speed of computation and economies the storage capacity of computers. The developed Continuous Element can be used efficiently for analyzing joined cross-ply laminated conical-cylindrical-conical shells containing fluid in medium and high frequencies where other current methods meet difficulties.

+ The fluid filling can reduce significantly the natural frequencies of thick joined cross-ply laminated conical-cylindrical-conical shells filled with fluid. Frequency reduction is shown to increase with liquid depth.

+ Natural frequency of composite joined cross-ply laminated conical-cylindricalconical shells containing fluid decreases as ratio $L_{1} / R_{1}$ increases. The reduction of natural frequencies of composite joined cross-ply laminated conical-cylindrical-conical shells is significant as mode $m=1$ or 2 and $n=1$.

The present Continuous Element model can be expanded to solve the vibration problem of joined composite conical-cylindrical-conical shell filled with fluid surrounded by an elastic foundation.

\section{ACKNOWLEDGMENTS}

This research is funded by Vietnam National Foundation for Science and Technology Development (NAFOSTED) under Grant number: 107.02-2015.14.

\section{REFERENCES}

[1] K. R. Sivadas and N. Ganesan. Vibration analysis of thick composite clamped conical shells of varying thickness. Journal of Sound and Vibration, 152, (1), (1992), pp. 27-37. doi:10.1016/0022460x(92)90063-4.

[2] Z. C. Xi, L. H. Yam, and T. P. Leung. Free vibration of a laminated composite circular cylindrical shell partially filled with fluid. Composites Part B: Engineering, 28, (4), (1997), pp. 359-374. doi:10.1016/s1359-8368(96)00047-9.

[3] Z. C. Xi, L. H. Yam, and T. P. Leung. Free vibration of a partially fluid-filled cross-ply laminated composite circular cylindrical shell. The Journal of the Acoustical Society of America, 101, (2), (1997), pp. 909-917. doi:10.1121/1.418049.

[4] O. Katsutoshi, T. Unji, and S. Mikio. Free vibrations of a laminated composite circular cylindrical shell partially filled with liquid. Transactions of the Japan Society of Mechanical Engineers C, 65, (640), (1999), p. 45974604. doi:10.1299/kikaic.65.4597.

[5] M. H. Toorani and A. A. Lakis. Shear deformation in dynamic analysis of anisotropic laminated open cylindrical shells filled with or subjected to a flowing fluid. Computer Methods in Applied Mechanics and Engineering, 190, (37), (2001), pp. 4929-4966. doi:10.1016/s00457825(00)00357-1. 
Vu Quoc Hien, Tran Ich Thinh, Nguyen Manh Cuong

[6] J. Kochupillai, N. Ganesan, and C. Padmanabhan. A semi-analytical coupled finite element formulation for composite shells conveying fluids. Journal of Sound and Vibration, 258, (2), (2002), pp. 287-307. doi:10.1006/jsvi.2002.5176.

[7] W. Larbi, J. F. Deü, and R. Ohayon. Vibration of axisymmetric composite piezoelectric shells coupled with internal fluid. International Journal for Numerical Methods in Engineering, 71, (12), (2007), pp. 1412-1435. doi:10.1002/nme.1987.

[8] M. H. Toorani and A. A. Lakis. Swelling effect on the dynamic behaviour of composite cylindrical shells conveying fluid. International Journal for Numerical Methods in Fluids, 50, (4), (2006), pp. 397-420. doi:10.1002/fld.1058.

[9] L. Tong. Free vibration of orthotropic conical shells. International Journal of Engineering Science, 31, (5), (1993), pp. 719-733. doi:10.1016/0020-7225(93)90120-j.

[10] L. Tong. Free vibration of composite laminated conical shells. International Journal of Mechanical Sciences, 35, (1), (1993), pp. 47-61. doi:10.1016/0020-7403(93)90064-2.

[11] C. Shu. An efficient approach for free vibration analysis of conical shells. International Journal of Mechanical Sciences, 38, (8), (1996), pp. 935-949. doi:10.1016/0020-7403(95)00096-8.

[12] Y. Narita, Y. Ohta, and M. Saito. Finite element study for natural frequencies of cross-ply laminated cylindrical shells. Composite Structures, 26, (1-2), (1993), pp. 55-62. doi:10.1016/02638223(93)90044-q.

[13] K. K. Viswanathan, K. S. Kim, J. H. Lee, H. S. Koh, and J. B. Lee. Free vibration of multilayered circular cylindrical shell with cross-ply walls, including shear deformation by using spline function method. Journal of Mechanical Science and Technology, 22, (11), (2008), pp. 20622075. doi:10.1007/s12206-008-0747-4.

[14] A. A. Khdeir, J. N. Reddy, and D. Frederick. A study of bending, vibration and buckling of cross-ply circular cylindrical shells with various shell theories. International Journal of Engineering Science, 27, (11), (1989), pp. 1337-1351. doi:10.1016/0020-7225(89)90058-x.

[15] D. S. Kumar and N. Ganesan. Dynamic analysis of conical shells conveying fluid. Journal of Sound and Vibration, 310, (1), (2008), pp. 38-57. doi:10.1016/j.jsv.2007.07.020.

[16] Y. Kerboua, A. A. Lakis, and M. Hmila. Vibration analysis of truncated conical shells subjected to flowing fluid. Applied Mathematical Modelling, 34, (3), (2010), pp. 791-809. doi:10.1016/j.apm.2009.06.028.

[17] T. Irie, G. Yamada, and Y. Muramoto. Free vibration of joined conical-cylindrical shells. Journal of Sound and Vibration, 95, (1), (1984), pp. 31-39. doi:10.1016/0022-460x(84)90256-6.

[18] B. P. Patel, M. Ganapathi, and S. Kamat. Free vibration characteristics of laminated composite joined conical-cylindrical shells. Journal of Sound and Vibration, 237, (5), (2000), pp. 920-930. doi:10.1006/jsvi.2000.3018.

[19] M. Caresta and N. J. Kessissoglou. Free vibrational characteristics of isotropic coupled cylindrical-conical shells. Journal of Sound and Vibration, 329, (6), (2010), pp. 733-751. doi:10.1016/j.jsv.2009.10.003.

[20] M. A. Kouchakzadeh and M. Shakouri. Free vibration analysis of joined cross-ply laminated conical shells. International Journal of Mechanical Sciences, 78, (2014), pp. 118-125. doi:10.1016/j.ijmecsci.2013.11.008.

[21] J. R. Banerjee and A. Sobey. Dynamic stiffness formulation and free vibration analysis of a three-layered sandwich beam. International Journal of Solids and Structures, 42, (8), (2005), pp. 2181-2197. doi:10.1016/j.ijsolstr.2004.09.013.

[22] J. R. Banerjee and F. W. Williams. Coupled bending-torsional dynamic stiffness matrix for timoshenko beam elements. Computers \& Structures, 42, (3), (1992), pp. 301-310. doi:10.1016/0045-7949(92)90026-v. 
[23] M. C. Nguyen. Elements continus de plaques et coques avec prise en compte du cisaillement transverse: application à l'interaction fluide-structure. PhD thesis, Universit Paris 6, (2003).

[24] J. B. Casimir, M. C. Nguyen, and I. Tawfiq. Thick shells of revolution: Derivation of the dynamic stiffness matrix of continuous elements and application to a tested cylinder. Computers E Structures, 85, (23), (2007), pp. 1845-1857. doi:10.1016/j.compstruc.2007.03.002.

[25] T. I. Thinh and M. C. Nguyen. Dynamic stiffness matrix of continuous element for vibration of thick cross-ply laminated composite cylindrical shells. Composite Structures, 98, (2013), pp. 93-102. doi:10.1016/j.compstruct.2012.11.014.

[26] M. C. Nguyen, T. I. Thinh, and T. T. Hien. Vibration analysis of thick laminated composite conical shells by CEM. In Proceedings of the 9th National Congress on Mechanics, Hanoi, (2012).

[27] T. I. Thinh, N. M. Cuong, and V. Q. Hien. Dynamic stiffness method for free vibrations analysis of partial fluid-filled orthotropic circular cylindrical shells. Vietnam Journal of Mechanics, 37, (1), (2015), pp. 43-56. doi:10.15625/0866-7136/37/1/5508.

[28] M. C. Nguyen, T. I. Thinh, and V. Q. Hien. Vibration analysis of cross-ply composite joined conical-cylindrical shells by continuous element method. In Proceedings of the International Conference on Engineering Mechanics and Automation-ICEMA3, Hanoi, (2014). pp. 401-408.

\section{APPENDIX}

Matrix $[A(\omega)]_{10 \times 10}$

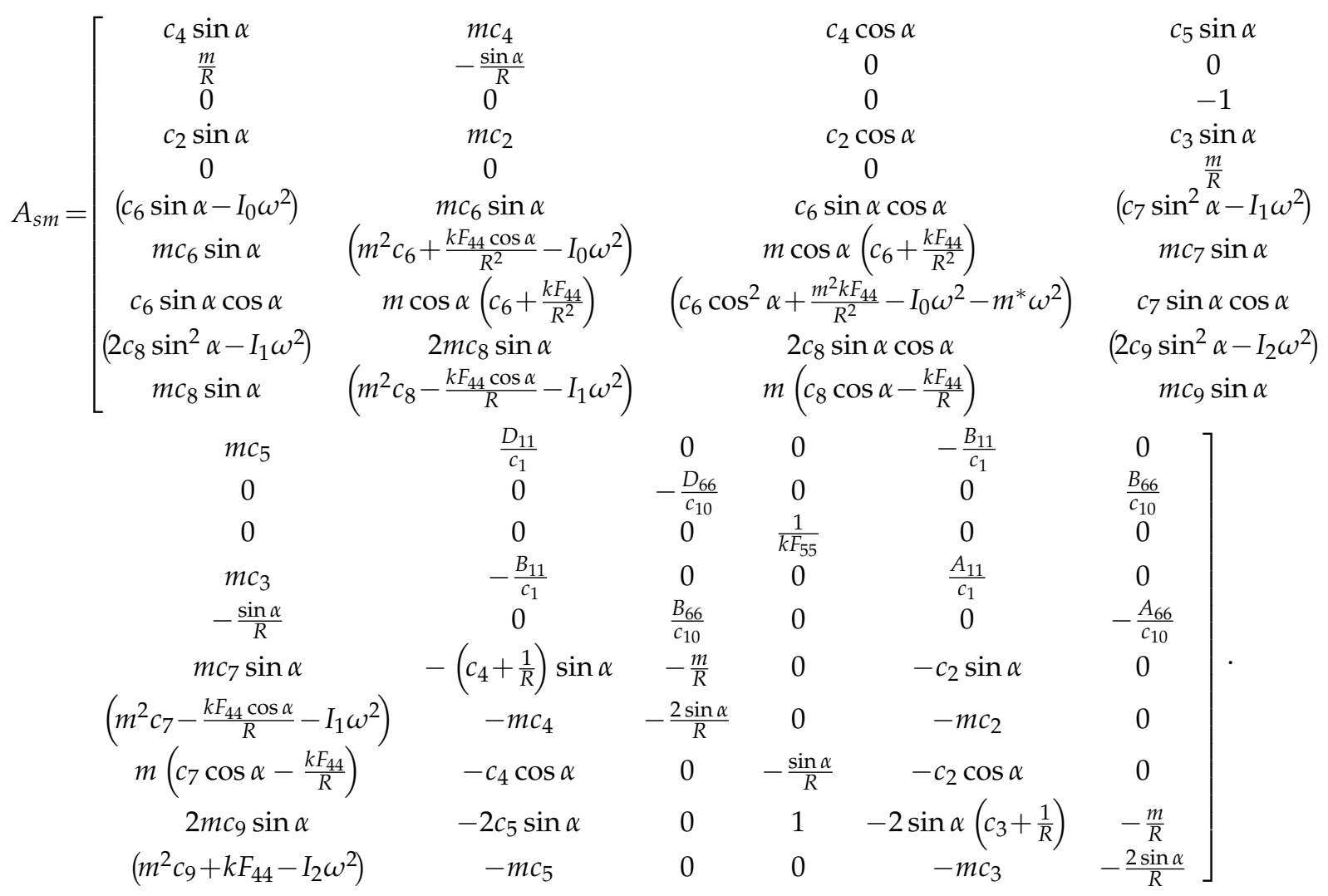

